\title{
Coma caused by Shigella toxin-producing Escherichia coli in the absence of haemorrhagic uraemic syndrome (HUS)
}

\author{
Kristien Velding ${ }^{1}$, Bert Mulder ${ }^{2}$, Jeroen Oeben ${ }^{1}$, Annelies Suurmeijer $^{1}$, Thiemo F Veneman $^{1}$ \\ ${ }^{1}$ Department of Intensive Care Medicine, Ziekenhuisgroep Twente (ZGT), Almelo, The Netherlands \\ ${ }^{2}$ Laboratorium Microbiologie Twente Achterhoek, Hengelo, The Netherlands
}

\section{Doi: 10.12890/2016_000418 - European Journal of Case Reports in Internal Medicine - @ EFIM 2016}

Received: $27 / 02 / 2016$

Accepted: $23 / 04 / 2016$

Published: 08/06/2016

How to cite this article: Velding K, Mulder B, Oeben J, Suurmeijer A, Veneman TF. Coma caused by Shigella toxin-producing Escherichia coli in the absence of haemorrhagic uraemic syndrome (HUS). EJCRIM 2016;3:doi:10.12890/2016_000418.

Conflicts of Interests: The Authors declare that there are no competing interests.

This article is licensed under a Commons Attribution Non-Commercial 4.0 License

\section{ABSTRACT}

Shigella toxin-producing Escherichia coli (STEC) is well known for its complications such as haemolytic uraemic syndrome (HUS), but neurological symptoms have also been reported. While most cases of infection with STEC occur with concurrent HUS, we describe a patient with severe neurological symptoms in the absence of HUS.

\section{LEARNING POINTS}

- Shigella toxin producing Escherichia coli (STEC) are bacteria that cause haemorrhagic colitis.

- Generally, infections with STEC occur with a concurrent haemolytic uraemic syndrome (HUS).

- However, infections with STEC can occur with neurological symptoms without HUS.

\section{KEYWORDS}

Hemolytic uraemic syndrome, Shigella toxin, Escherichia Coli.

\section{INTRODUCTION}

Shigella toxin-producing Escherichia coli (STEC) is well known for its complications such as haemolytic uraemic syndrome (HUS), but neurological symptoms have also been reported. Recent studies on STEC epidemics report a high prevalence of neurological symptoms with a rapid degenerative course, often requiring intubation and admission to the intensive care unit ${ }^{[1-5]}$. While most of these cases occur with concurrent HUS, we describe a patient with severe neurological symptoms due to infection with STEC in the absence of HUS. We would like to emphasize that infection with STEC should be included in the differential diagnosis when neurological symptoms and gastro-enteritis occur together.

\section{CASE PRESENTATION}

A 79-year-old woman was admitted to the internal medicine ward with a 7-day history of fever, vomiting, abdominal pain and diarrhoea. She was empirically treated with amoxicillin by her general physician.

On presentation she did not appear ill and had a body temperature of $38.1^{\circ} \mathrm{C}$, blood pressure of $134 / 72 \mathrm{mmHg}$ and $\mathrm{SpO}_{2}$ of $97 \%$ on room air. Physical examination was unremarkable except for slight tenderness in the right upper quadrant of the abdominal region on palpation. Laboratory tests showed increased C-reactive protein (CRP; $30 \mathrm{mg} / \mathrm{l}$, normal $<10 \mathrm{mg} /$ ) and slightly decreased renal function (creatinine 116 
$\mu \mathrm{mol} / \mathrm{ml}$, eGFR 39). Arterial blood gas analysis, urea, electrolytes, plasma glucose and urine dipstick showed no abnormalities.

A CT scan of the abdomen showed increased vascularization and thickening of the colon wall.

During the following days the patient developed headaches and became progressively confused, followed by a reduced level of consciousness. She also showed generalized weakness and a tremor. There were no signs of neck stiffness or focal neurology. The patient then had a seizure and after administration of clonazepam became comatose and developed respiratory insufficiency. She was therefore admitted to the intensive care unit for mechanical ventilation. Cerebrospinal fluid analysis showed an elevated leucocyte count (15.3×106/l, normal $<5 \times 106 / \mathrm{l})$ and raised total protein $(1295 \mathrm{mg} / \mathrm{l}$, normal $250-800 \mathrm{mg} / \mathrm{l})$. The CRP and leucocyte count continued to increase during antibiotic therapy with intravenous ceftriaxone $2 \mathrm{~g}$ daily and intravenous acyclovir $10 \mathrm{mg} / \mathrm{kg}$ every $8 \mathrm{~h}$. A CT scan of the cerebrum showed no abnormalities. Cultures and PCR analysis of cerebrospinal fluid were negative for common viruses and bacteria causing meningitis or encephalitis (herpes simplex virus 1 and 2, Varicella zoster virus, Haemophilus influenzae, Mycoplasma pneumoniae, Neisseria meningitidis, human Parechovirus, Streptococcus agalactiae, Streptococcus pneumoniae), as well as for enterovirus, adenovirus and Mycobacterium tuberculosis. Molecular diagnostic assay on faecal matter for common pathogens causing gastro-enteritis yielded enterohaemorrhagic E. coli with an unidentified serotype. MRI of the brain showed periventricular and subcortical white matter lesions (Fig. 1), as well as discrete bilateral white matter changes in the capsula externa and globus pallidus. Syphilis, Borrelia burgdorferi, HIV, EBV and CMV serology was negative; regular blood and urine cultures were also negative.

Based on the clinical presentation and findings of additional investigations, we ruled out electrolyte and metabolic abnormalities, trauma, and structural or vascular cerebral pathology. A diagnosis of STEC infection with Shigella toxin-associated neurological impairment was established.

The patient remained on mechanical ventilation for 7 days. On the fifth day after admission to the intensive care unit, she showed the first signs of neurological improvement: her level of consciousness fluctuated, and high reflexes and a trismus were observed. Gradually, neurological symptoms subsided and after 10 days the patient was discharged from the intensive care unit in good clinical condition.

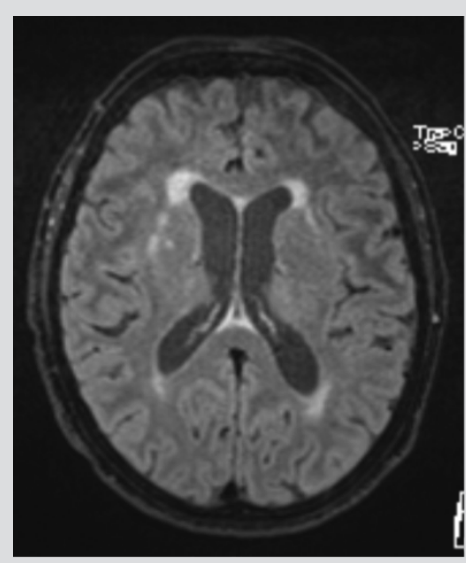

Figure 1. MRI of the brain showing periventricular and subcortical white matter lesions, as well as discrete bilateral white matter changes in the capsula externa and globus pallidus.

\section{DISCUSSION}

STEC are bacteria that cause haemorrhagic colitis with potentially severe Shigella toxin-related complications manifesting as HUS and neurological symptoms. STEC is transmitted through contaminated food, is seen primarily in children and occurs mainly in epidemics. However, adults are also affected and isolated cases do occur. The yearly number of stool-confirmed reported cases in the Netherlands has risen from approximately 50 cases in 2006 to 1,173 cases in 2013, a remarkable increase in incidence that can partly be attributed to the introduction of new detection techniques such as PCR and better registration of cases. However, incidence is unequally distributed among O-157, non-157 and non-identified serotypes, with the largest increase in cases involving unidentified serotypes ${ }^{[1]}$. This suggests an actual increase in the incidence of STEC and involvement of previously uncommon serotypes.

In 2011 an outbreak of STEC occurred in Western Europe, with around 3,000 cases of Shigella toxin-mediated gastro-enteritis reported in Germany alone. While HUS is a common complication of STEC, studies based on the 2011 outbreak reported neurological symptoms in unusually high numbers of patients $(26-48 \%)^{[2-4]}$. Reported symptoms included tremors, seizures and neuropsychiatric disorders. A 
prospective single centre study ${ }^{[3]}$ carried out among 61 confirmed STEC patients reported that neurological symptoms were seen in $43 \%$ of these patients, of whom $50 \%$ had seizures and $34 \%$ had tremors. Furthermore, nearly all cases involving neurological symptoms were rapidly progressive, with symptoms deteriorating within hours towards complex syndromes. A study of 217 patients with STEC reported that $33 \%$ of patients with neurological symptoms required intubation and assisted ventilation on the intensive care unit ${ }^{[4]}$. This rate was significantly higher than that in a group of patients who only had STEC-HUS.

Whereas isolated HUS in STEC without neurological symptoms is common, neurological symptoms complicating STEC infection without HUS are rare, but were nevertheless reported in 3/61 and 4/104 patients, respectively, in the above-mentioned studies ${ }^{[3,4]}$. Interestingly, most of these neurological symptoms were caused by the O-104 enterohaemorrhagic E. coli serotype. This suggests that there may be an association between unique clinical features and different bacterial strains. Also, since the pathophysiology of Shigella toxin-related end organ injury is not completely understood, it might differ between kidney and brain ${ }^{[5]}$.

The diagnosis of HUS relies primarily on laboratory findings, but the diagnosis of neurological symptoms related to STEC is mainly based on the clinical presentation combined with imaging studies with a preference for MRI. Lesions on T2-weighted and diffusion-weighted MRI reported in the literature include symmetrical hyperintensities in the region of the sixth cranial nerve, the thalamus, and juxtacortical and periventricular white matter ${ }^{[2,4]}$. Generally, only supportive treatment is given for STEC-associated encephalopathy ${ }^{[2,4]}$.

Several studies have suggested eculizumab as an experimental treatment ${ }^{[4,5]}$. Although STEC-related neurological symptoms increase the risk of mortality, they are usually transient in those who survive.

A remarkable aspect of the described case is that it was an isolated case of STEC-associated encephalopathy, without a determined source of infection. To our knowledge this is the first case reported in the absence of an epidemic. We would like to stress that STEC-mediated encephalopathy is a diagnosis of exclusion, but with the increasing incidence of STEC, Shigella toxin-related brain injury should be included in the differential diagnosis in cases of gastro-enteritis and unexplained neurological symptoms.

\section{REFERENCES}

1. Friesema IHM, Kuiling S, Biesta-Peters EG, Zuidema R, van der Zwaluw WK, Heck MEOC, et al. Surveillance van shigatoxineproducerende Escherichia coli (STEC) in Nederland 2013. http://www.rivm.nl/Documenten_en_publicaties/Algemeen_Actueel/Uitgaven/Infectieziekten_Bulletin/Jaargang_26_2015/Februari_2016 [in Dutch].

2. Wegenroth M, Hoeltje J, Repenthin J, Meyer TN, Bonk F, Becker H, et al. Central nervous system involvement in adults with epidemic hemolytic uremic syndrome. AJNR Am J Neuroradiol 2013;34:1016-1021.

3. Ullrich S, Bremer P, Neumann-Grutzeck C, Otto H, Rüther C, Uwe von Seydewitz C, et al. Symptoms and clinical course of EHEC O104 infection in hospitalized patients: a prospective single center study. PLoS One 2013;8:e55278.

4. Magnus T, Röther J, Simova O, Meier-Cillien M, Repenthin J, Möller F, et al. The neurological syndrome in adults during the 2011 northern German E. coli serotype O104:H4 outbreak. Brain 2012;135(Pt 6):1850-1859.

5. Trachtman H, Austin C, Lewinski M, Stahl RAK. Renal and neurological involvement in typical Shiga toxin-associated HUS. Nat Rev Nephrol 2012;8:658-669. 